\title{
EARLINET APPROACH TO OPTIMISATION OF INDIVIDUAL NETWORK INSTRUMENTS WITH THE AIM OF HOMOGENISATION OF AEROSOL DATA PRODUCTS AND INCREASED DATA COVERAGE
}

\author{
Arnoud Apituley* (RIVM, Bilthoven, The Netherlands) \\ Volker Freudenthaler (University of Munich, Germany) \\ Adolfo Comerón, Francesc Rocadenbosch (Universitat Politècnica de Catalunya (UPC, Barcelona, \\ Spain)
}

\begin{abstract}
The European Aerosol Research LIdar NETwork (EARLINET) is an aerosol lidar network on a continental scale. EARLINET is now a leading network in qualitycontrolled quantitative aerosol profiling performing a schedule of routine measurements and presently consists of 28 stations distributed over Europe. The construction of an un-biased spatio-temporal database of vertical profiles of aerosol optical properties on a regional scale for climate and air quality research is the main objective of EARLINET and is accomplished through application of Raman lidars. One of the tasks in the EC-funded project EARLINET-ASOS is to optimize individual instruments with the aim of homogenization of aerosol data products over the network and increased data coverage by automation. This task is approached by selection of optimal solutions existing in the pool of individual stations. This is done for components, subsystems as well as for system integration. In system integration emphasis lies on automation to reduce the amount of manpower needed, to improve temporal coverage, and to make performance independent from individual operators. The procedure to perform these tasks is outlined and the set of tools enabling the assessment of performance under development is described.
\end{abstract}

Index Terms - Lidar, network, aerosol, harmonization, automation

\section{INTRODUCTION}

Among the factors causing the largest uncertainties in the understanding of climatic change are aerosols and cloud feedback mechanisms involving the role of aerosols. This is due to the variable nature in space and time of the aerosols, that are relatively short-lived atmospheric species, but can still be distributed over large distances when the conditions are right. Aerosols and their climatic impact are therefore much more difficult to catch in atmospheric models than long-lived greenhouse gases such as carbondioxide. Better knowledge of the spatio-temporal distrubution of aerosols is critically needed to better assess their role in climate change [1].

The European Aerosol Research LIdar NETwork (EARLINET) started in 2000 under the EC 5th Framework Program. The network presently consists of 28 stations distributed over Europe (from Norway to Spain and from Ireland to Georgia). The construction of an un-biased spatiotemporal database of vertical profiles of aerosol optical properties on a regional scale for climate and air quality research is the main objective of EARLINET. This cannot be accomplished by backscatter lidars since these do not provide quantitative optical aerosol data without critical assumptions. Therefore, EARLINET aims to apply mainly Raman lidars that are quantitative.

The increasing number of participating stations illustrate the success of this initiative. However, in order to build the aerosol database, rigorous quality assurance is needed and also homogeneous data quality is desired so that the network can be used truly as a single instrument. Most stations in the network have lidars that were not series-produced and consequently these instruments have differences in set-up and construction. Consequently, the performance of the individual instruments may be different, for instance with respect to the altitude range covered or the number of operating wavelengths.

From 2006 until 2011, the network's development is supported by the EC-project EARLINET- ASOS (Advanced Sustainable Observation System). The main objective of this Coordination Action is to improve the EARLINET infrastructure for better spatial and temporal coverage of the observations, continuous quality control for the complete observation system, and fast delivery of standardized data products. One of the tasks in EARLINETASOS is to optimize individual instruments with the aim of homogenization of aerosol data products over the network and increased data coverage by automation.

This will be accomplished by selecting the optimal approach from the various solutions existing at individual stations for components and subsystems as well as for system

\footnotetext{
${ }^{*}$ RIVM - National Institute for Public Health and the Environment, P.O. Box 1, NL3720BA Bilthoven, The Netherlands. Email: Arnoud.Apituley@rivm.nl.
} 
integration. In system integration emphasis is on automation of operation to reduce the amount of manpower needed, to improve temporal coverage, and to make performance independent from individual operators.

\section{HANDBOOK OF INSTRUMENTS}

The EARLINET approach for optimization and harmonization of the instrument performance across the network is starts with an extensive list of system parameters that all stations' instrument PI's are required to complete: the Handbook of Instruments (HoI). The basis of the structure of the HoI is a generic lidar system, such as depicted in Fig.1. The HoI's structure can accommodate a wide variety of system configurations that can still be evaluated using exactly the same tools. The HoI allows for multiple emitted wavelengths, from one or more lasers. The detection chain is set up for each detected wavelength separately and includes parameters to adequately describe the properties of the detection channels in terms of e.g. spectral response, detector efficiency, polarisation properties, etc. The total number of parameters for a single wavelength backscatter lidar is about 100. Station characteristics, including accessibility of ancillary data such as radiosondes and sunphotemeters are also included.

In this way the HoI thoroughly documents each EARLINET station and serves as an inventory of existing technical solutions in the network for quantitative aerosol profiling and as a characterization of the station.

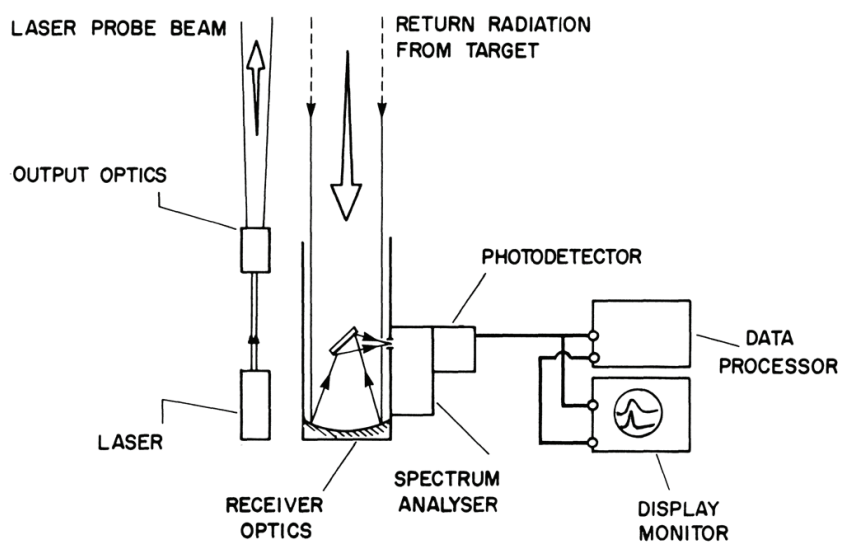

Fig.1. Generic set-up of a lidar system with a laser emitter, a receiving telescope, wavelength-selection, a light sensitive detector and recording electronics.

The HoI will be used in the following way:

- Assess conceptual performance and performance limits of lidar systems by using the configuration data provided to model (parts of) individual systems. This can be done, for instance, for an isolated detection channel (i.e. one detected wavelength) in a particular lidar. In this way, it becomes possible to compare lidar systems' characteristics among each other and reduce the necessity for direct instrument intercomparisons.

- The handbook provides a detailed overview of possible configurations of lidars for aerosol profiling that will be studied to find optimized solutions. This is particularly helpful to new groups that will be able to benefit from existing knowledge.

- The handbook will be studied to find the more common problems where joint efforts are expected to yield effective results on the improvement on the over-all quality of the data provided by the network.

The HoI is set up as 'live' documentation on the web and is accessible through the internal EARLINET web-pages. Since it is expected that the instruments in the network to be improved upon during the course of the project, all groups are requested to update the information on their configuration changes as soon as it is used for production of data submitted to the database. Older configurations, used for data production in the past, can still be retrieved after newer configurations have taken effect.

\section{INSTRUMENT OPTIMIZATION AND HARMONIZATION}

Optimization and harmonization of the instrument performance given the wide variety of instruments in the network is a task not to be underestimated. The number of instrumental details to be addressed can become very large, considering the wide variety of issues and lidar systems involved. Therefore, the activity started looking for optimizations fitting the following overall goals:

- Optimal performance for the main data products of our lidars for aerosol pro-filing: i.e. aerosol backscatter profiles, aerosol extinction profiles and lidar ratio. The minimum recommendation for an optimized product is to add a nitrogen Raman channel to a backscatter lidar.

- Daytime performance.

Aerosol radiative properties play their most important role during daytime. Aerosol characteristics can change in relatively short periods of time. The lidar ratio obtained during nighttime measurements can differ significantly from that actually occurring in aerosols whose elastic backscatter is measured during daytime hours before or after. Therefore daytime measurements of the aerosol properties are needed and technical solution to accomplish this.

- Increased operationality

Increase automation in instrument control so that more data will become available, at a reduced manpower/data ratio. This will be treated separately.

The overall optimization and harmonization requires 1) a set of tools that are used to evaluate actual system performance based on the data provided in the HoI, and 2) a set of requirements for the EARLINET database and 3) a set of hardware tests to diagnose instrumental problems. Where 
discrepancies between 1) and 2) exist, further tests with 3) may be used to indicate malfunctioning or badly configured hardware. Sub-optimal configurations can be identified.

The general approach can be broken down in the following iterative sequence:

1. Collect essential system information and compile it in the handbook of in-struments (HoI)

2. Define criteria for system performance, according to requirements of the EARLINET database (i.e. maximum error of aerosol properties vs height. These requirements will be classified in a number of categories. Suggested are PBL, PBL+ residual layers, Upper free troposphere)

3. Develop and apply tools to assess individual system performance, based on data in the HoI, and perhaps system intercomparisons

4. Check the system performance against the requirements in 2 .

5. If errors are found to be outside the requirements, optimizations are needed

6. HoI can be used to find improvements and implement them. Perhaps new technologies need to be developed.

7. Update HoI

8. Re-iterate from 4

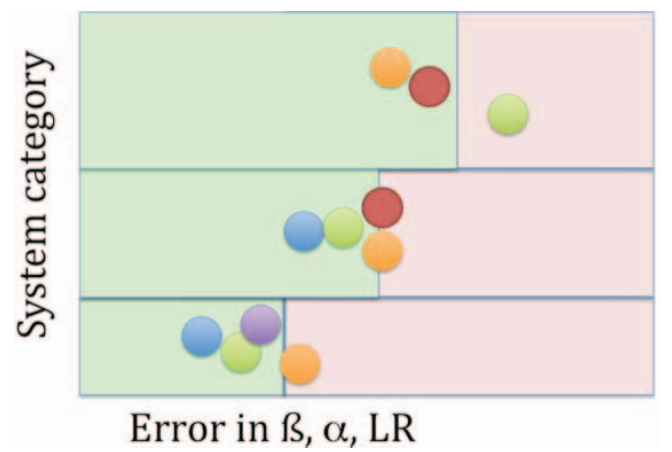

Fig.2 Graph schematically indicating system performance. Each colored circle represents a lidar system, that may appear in more than one category (e.g. PBL range coverage, free tropospheric range coverage, high performance multiwavelength system). After optimization, all systems should migrate into the acceptable region to the left.

Specific tools that are used to assess system performance can be split between tools dealing with the optical configuration of the system and tools dealing with the electrical sub-systems and data acquisition electronics.

For the optical part, ray-tracing techniques can be applied to test theoretical performance against practice. Experimental tests have also been developed to look at the properties of optical sub-systems. For instance, when the different parts of the collecting area of the receiving telescope are covered during test measurements, intricately hidden problems in the receiver may be revealed [2].
From the point of view of the signal processing, tools are currently underdevelopment that convert the data in the HoI into dimensionless parameters that can be used to assess performance limits on a per-channel basis, beyond the simple power-aperture product. [3]

Recently, inversion algorithms have been developed to obtain microphysical aerosol properties such as effective radius, volume and surface-area concentration, real and imaginary part of the complex refractive index, and singlescattering albedo from multi-wavelength Raman lidar observations. Backscatter coefficients at three wavelengths $(355,532,1064 \mathrm{~nm})$ plus extinction coefficients at two wavelengths $(355,532 \mathrm{~nm})$ are the minimum required input data for such inversion schemes [4]. Presently, eight EARLINET stations perform at this level. EARLINET strives to further increase the number of stations equipped with such high-performance instruments and the approach for optimization and tools presented here are expected to play an important part in this. If our approach is successful, also, future expansion of EARLINET data products can be instrumentally optimized in this way.

More and more frequently, new data products are requested from the network, such as cloud data. These new data products also require specific optimizations of the lidar systems. The optimization and harmonization activity in EARLINET-ASOS will play a role in this.

\section{SYSTEM INTEGRATION}

System integration is a separate subject, since it is not directly related to the scientific data products that are produced, but proper integration as well as automation, can have a tremendous impact on reliability and operability of an instrument. So proper integration is a prerequisite to steady and reliable dataflow and to make performance independent from individual operators.

To clarify what can be done at various levels of system integration and automation, the generic lidar is broken down in sub-systems. For each sub-system a range of possibilities is given for integration, taking into account mechanical, optical, electronic and remote control issues. An inventory of requirements and solutions are per sub-system is compiled in a structure starting from sub-systems to lager units:

- Integration, e.g.

- Mechanical stability

- Serviceable

- Modular

- Upgradable

- Electrical noise control

- Automation of Sub-Systems, e.g.

- Overlap control

- Laser power stabilization

- Adjustable signal dynamic range

- Housing, e.g.
- Climatisation
- Closed window 
Tab.1 Example of a Handbook of Instruments table for a Raman lidar with a three wavelength laser and three separate receiving telescopes for close range, far range and polarisation measurements.

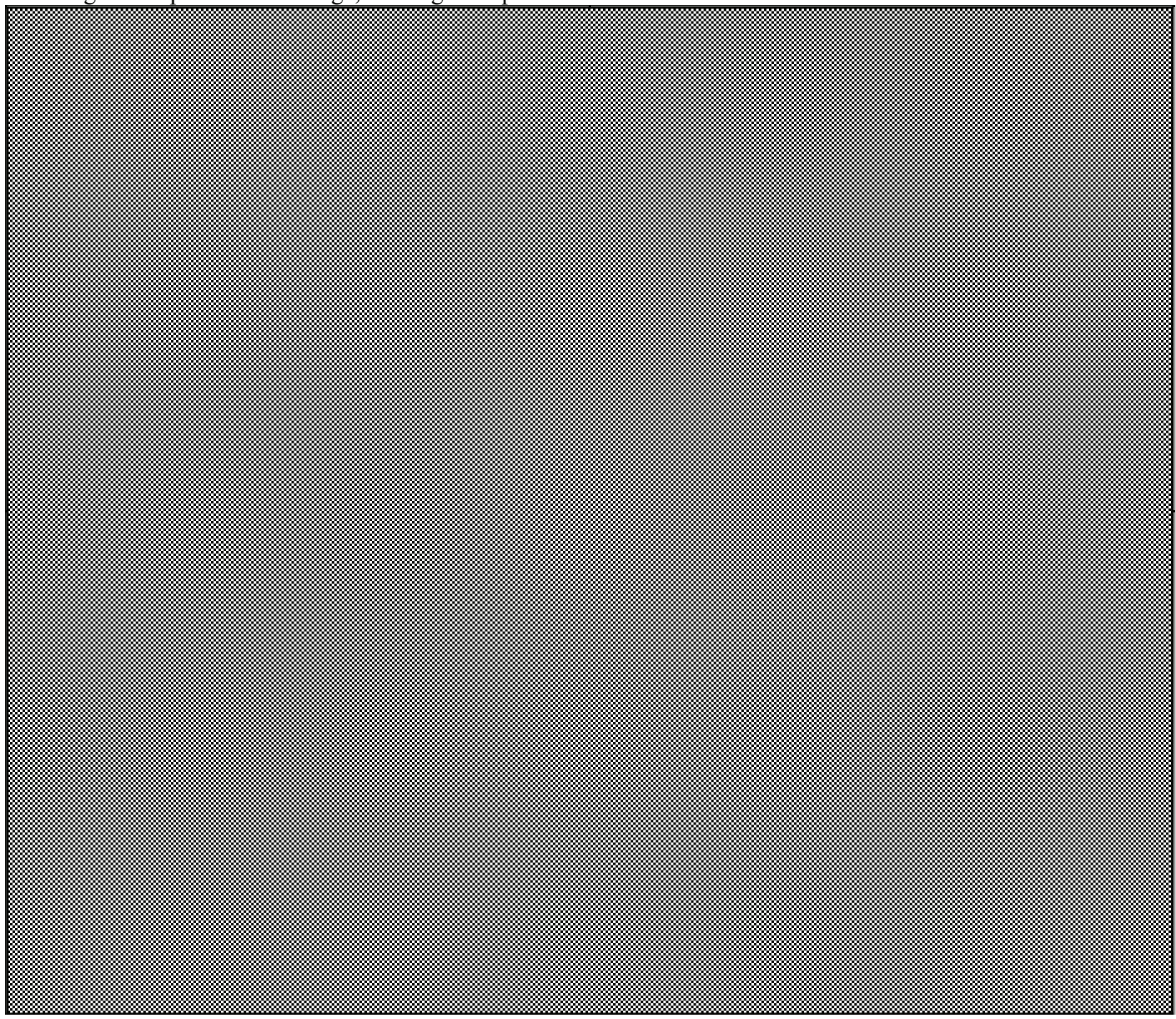

- Unattended Operation, e.g.

$\circ$ Automatic configuration (signal dynamic range)

- Monitor signals and dynamically make adjustments.

- Eye-safety/aircraft protection

- Unattended remote operation

○ Remote access to the system and all subsystems

It may not be possible or even necessary to jump from a manually operated lidar to a fully remotely controllable system. Rather than this, proper automation of a critical subsystem, such as adjustment of overlap between reveiving telescope and laserbeam, may be an effective way to improve data reliability. By subsequently automating parts of the lidar (automation will require funds to purchase hardware and manpower to build software) a better integrated and automated system will be come available.

\section{ACKNOWLEDGEMENT}

EARLINET-ASOS is funded by the European Commission under grant RICA-025991.

\section{REFERENCES}

[1] Diner, D. J., et al. (2004), PARAGON: An Integrated Approach for Characterizing Aerosol Climate Impacts and Environmental Interactions, Bulletin of the American Meteorological Society, 85(10), 1491-1501.

[2] Freudenthaler, V., The Telecover Test: A quality assurance tool for the optical part of a lidar system, Proceedings ILRC24, Boulder, Colorado, June 22-27, 2008.

[3] R. Agishev et al: "Lidarperformance prediction by dimensionless SNR-parameterization”, Proc. of SPIE Vol. 5979 59791G-1 G-10, doi 10.1117/12.627569

[4] Müller, D., et al. (1999), Microphysical particle parameters from extinction and backscatter lidar data by inversion with regularisation: theory, Applied Optics, 38(12), 2346-2357. 